\title{
Data on the Orthoptera assemblages of characteristic agricultural landscape in the Carpathian Lowland
}

\author{
Dóra Arnóczkyné Jakab - Antal Nagy \\ University of Debrecen, Institute of Plant Protection, H-4032 Debrecen, Böszörményi út 138. \\ jakidori6@gmail.com
}

\begin{abstract}
SUMMARY
Orthoptera fauna and assemblages of natural and semi-natural grasslands of the Hungarian Lowland are well-known, however, little is known about assemblages living in agricultural and anthropogenic habitats such as arable lands, roadsides, hedges, and riverbanks. Due to climate change, intensification of agriculture, and change of habitat use, these habitat types become increasingly important.

To collect data on these mainly unknown habitat types, a three-year study was carried out on the Orthoptera fauna and assemblages of the firth region of the Tisza and Sajó rivers. This area was mainly unknown, and our research contributes to increasing knowledge and provides a base for further investigations.

In the 40 sampling sites of the studied region, an occurrence of 30 Orthoptera species was recorded based on 2241 sampled individuals. In this study, we provide 540 new distribution data records of orthopterans that means an almost eightfold increase of the known data. Orthoptera assemblages of different agricultural habitat types showed significant differences considering both species richness and composition. Data suggested that non-cultivated habitat patches of dirt-roads, roadsides and stubble fields and even extensively used pastures, hayfields and alfalfa, red clover, and even wheat fields can preserve relatively species-rich Orthoptera assemblages. Contrary weedy sites of these cultivars and intensively used arable lands (maize, sunflower and rape fields) showed extremely low species diversity.
\end{abstract}

Keywords: Orthoptera; faunistical data; lowland; agriculture; distribution; species composition

\section{INTRODUCTION}

The intensification of agriculture and increasing use of pesticides started at the beginning of the $20^{\text {th }}$ century led to fragmentation, isolation, and habitat and biodiversity loss of natural and even semi-natural landscape (Foley et al., 2011; CBD, 2014; Batáry, 2018). The human population growth, consumption and quality requirements of customers present a severe problem to agriculture. The human population will increase by $30 \%$ while in the case of the food demand, this value will increase by $70 \%$ by 2050 (Conforti, 2011; Fróna, 2018). In Hungary, the efficiency of agriculture should be developed since the cultivated area can be expanded by up to 5\% (Agrárgazdasági Kutató Intézet, 2013). Therefore, the negative effect of this further intensification must be compensated with the intensification of ecosystem services and landscape protection (IPBES, 2016).

Changes of mixed agricultural and natural landscape can be monitored with species assemblages which are especially sensitive and can indicate the changes of their environment with changes of their qualitative and quantitative composition (Wiens, 1989; Pearson, 1994; McGeoch, 1998). Orthopterans are such kind of indicators considering their easy identification and sampling and relatively high species diversity. They are widely used in ecological and conservation biological studies mainly in grasslands and other opened habitats (Báldi and Kisbenedek, 1997; Kisbenedek, 1997; Batáry et al., 2007; Báldi et al., 2013; Torma et al., 2014; Kenyeres et al., 2020). Their diversity also well indicates the general species richness of their habitat thus their data can be used in conservation planning and decision-making (Noss, 1990).
Orthoptera fauna and assemblages of protected areas, natural and semi-natural habitats, and different project areas are relatively well known in Hungary (Nagy and Rácz, 2007). Arable- and wastelands and other anthropogenic habitats are poorly known due to the lack of sufficient studies conducted on these habitats (Nagy, 1953; Koppányi, 1957; Nagy, 1992; Nagy, 1993; Nagy et al., 2009) and in other sources only scattered data can be found (Nagy, 1943; ZilahiSebess, 1956; Garai, 1995; Báldi and Kisbenedek, 1997; Kenyeres et al., 2004; Kenyeres, 2006; Kenyeres, 2010; Szövényi et al., 2010; Kenyeres and Rácz, 2011). However due to agricultural intensification the importance and ecological functions of these artificial habitats are increasing day by day (Agócs et al., 2015). Barnabás Nagy already in the 1950s wrote about the unfavourable effect of stubble cultivation and decreased habitat diversity on the orthopterans of agricultural landscapes (Nagy, 1953). Although the intensive tillage also decreases diversity and abundance of Orthoptera assemblages (Koppányi, 1957), it has an important role in pest management (Arnóczkyné et al., 2020).

Orthoptera species show different sensitivity to various methods and intensity of cultivation. Change of intensity, methods, and even the crop rotation in a given site can dramatically change the composition of the Orthoptera assemblages and in some cases it can lead to local extinction of many sensitive and vulnerable species (Nagy, 1992, Kenyeres, 2006).

Survey and systematic monitoring of Orthoptera assemblages living in agricultural landscape is an important task in the point of view of both nature conservation and pest management. In our study, the first steps of such investigation were taken in the firth 
region of Sajó and Tisza Rivers between 2018 and 2020 and here we present the first results of this study.

\section{MATERIALS AND METHODS}

\section{Sampling sites}

Orthoptera assemblages of 40 sampling sites in the firth region of Sajó and Tisza rivers were investigated in a 3-year study between 2018 and 2020 (Figure 1). Four sampling sites $(2,8,18,26)$ were studied only in 2018 (Table 1). Samplings were carried out twice a year (in summer and early autumn) in the surroundings of Tiszaújváros, Sajószöged, Sajóörös, and Kesznyéten. Characteristic agricultural landscape elements (habitat types) were studied such as stubble-lands, pastures, hayfields, dirt-roads, roadsides, ruderal sites and maize, sunflower, wheat, alfalfa, and red clover fields.

\section{Data collection and samplings}

Sampling was carried out with a sweep net $(45 \mathrm{~cm}$ in diameter) done 200 sweeps in each sampling site. The net was emptied after 100 sweeps to protect the sampled specimens. The caught orthopterans were soon released after identification in the fields. Sweep net samplings were completed with 10 minutes direct search. In the habitats where sweep net could not be used (maize and sunflower fields and in tall, dense vegetation) only extended (30-40 minutes) direct search was used. Base on their different selectivity and effectiveness, these two methods well complete each other considering both quantitative and qualitative features of assemblages (Nagy et al., 2007).

Sampling sites were in the EU00 and EU01 $10 \times 10$ $\mathrm{km}$ UTM cells. To compare the sampled fauna with known assemblages of the surroundings, previously published data of these and neighbouring UTM cells (DU90, DU91, EU00, EU01, EU10 and EU11) were collected. In this case, actualized database of Nagy and Rácz (2007) was used. Till now only 4 articles (listed below) were published on the Orthoptera fauna of the studied region and its surroundings.

We built a database from newly collected and previously published distribution data. In case of published data, the following source codes were used:

[G] = Garai, A. (1995): Adatok Magyarország Orthoptera faunájához. Folia Entomologica Hungarica. 56: 231234.

[J] = Jablonowski, J. (1910): A nagy hortobágyi sáskairtás eredményei. Természettudományi Közlöny. 42 (509): 513-525.

[N] = Nagy, A.-Bozsó, M.-Kisfali, M.-Rácz, I. A. (2008): Data on the Orthoptera fauna of the Tisza district. Tiscia, Vegetation and Fauna of River Tisza Basin II: 1-22.

[R] = Rácz, I.A.-Varga, Z. (1978): Beitrage zur Kenntnis der Orthopteren-Fauna des Sandgebietes bei Igrici. Acta Biologica Debrecina. 15: 33-39.

The distribution data are presented in this paper as follows: code of sampling site (see Tables 1 and 2) / date of sampling or publication, habitat type (if it is known), source code (in case of published data).
In case of nomenclature, the Orthoptera Species File online database was followed (Orthoptera Species File, 2020).

Table 1. List of the 40 sampling sites of the Orthoptera assemblages investigated during the 3-year study between 2018 and 2020 in the firth region of the Sajó and Tisza rivers

\begin{tabular}{|c|c|c|c|c|c|c|}
\hline \multirow{2}{*}{$\begin{array}{l}\text { Sampling } \\
\text { site }\end{array}$} & \multirow{2}{*}{ GPS: N } & \multirow{2}{*}{ GPS: E } & \multirow{2}{*}{$\begin{array}{c}\text { UTM } \\
(10 \times 10 \mathrm{~km})\end{array}$} & \multicolumn{3}{|c|}{ Habitat } \\
\hline & & & & 2018 & 2019 & 2020 \\
\hline 1 & $47^{\circ} 56^{\prime} 32.70^{\prime \prime}$ & $21^{\circ} 1^{\prime} 58.99^{\prime \prime}$ & EU00 & stubble & maize field & stubble \\
\hline 2 & $47^{\circ} 56 ' 29.81^{\prime \prime}$ & $21^{\circ} 2^{\prime} 3.82^{\prime \prime}$ & EU00 & dirt-road & & \\
\hline 3 & $47^{\circ} 56^{\prime} 37.48^{\prime \prime}$ & $21^{\circ} 1^{\prime} 50.60^{\prime \prime}$ & EU01 & sunflower field & rape field & maize field \\
\hline 4 & $47^{\circ} 56^{\prime} 41.33^{\prime \prime}$ & $21^{\circ} 1^{\prime} 44.57^{\prime \prime}$ & EU01 & stubble & stubble & stubble \\
\hline 5 & $47^{\circ} 56^{\prime} 47.97^{\prime \prime}$ & $21^{\circ} 1^{\prime} 43.94^{\prime \prime}$ & EU01 & alfalfa field & wheat field & sunflower field \\
\hline 6 & $47^{\circ} 56^{\prime} 46.87^{\prime \prime}$ & $21^{\circ} 1^{\prime} 40.65^{\prime \prime}$ & EU01 & dirt-road & dirt-road & dirt-road \\
\hline 7 & $47^{\circ} 56^{\prime} 14.40^{\prime \prime}$ & $21^{\circ} 0 ' 21.68^{\prime \prime}$ & EU01 & dirt-road & dirt-road & dirt-road \\
\hline 8 & $47^{\circ} 56^{\prime} 13.31^{\prime \prime}$ & $21^{\circ} 0 ' 23.96^{\prime \prime}$ & EU00 & wheat field & & \\
\hline 9 & $47^{\circ} 56^{\prime} 23.31^{\prime \prime}$ & $21^{\circ} 0^{\prime} 27.29^{\prime \prime}$ & EU00 & dirt-road & dirt-road & dirt-road \\
\hline 10 & $47^{\circ} 56^{\prime} 22.47^{\prime \prime}$ & $21^{\circ} 0^{\prime} 29.23^{\prime \prime}$ & EU00 & stubble & maize field, stubble & maize field \\
\hline 11 & $47^{\circ} 56^{\prime} 21.11^{\prime \prime}$ & $21^{\circ} 0^{\prime} 27.52^{\prime \prime}$ & EU00 & stubble & wheat field & sunflower field \\
\hline 12 & $47^{\circ} 56^{\prime} 26.56^{\prime \prime}$ & $21^{\circ} 0^{\prime} 30.71^{\prime \prime}$ & EU00 & stubble & maize field & sunflower field \\
\hline 13 & $47^{\circ} 56^{\prime} 46.74^{\prime \prime}$ & $21^{\circ} 0^{\prime} 33.00^{\prime \prime}$ & EU01 & alfalfa field & alfalfa field & alfalfa field \\
\hline 14 & $47^{\circ} 56^{\prime} 48.15^{\prime \prime}$ & $21^{\circ} 0^{\prime} 35.66^{\prime \prime}$ & EU01 & stubble & wheat field & maize field \\
\hline 15 & $47^{\circ} 56^{\prime} 47.18^{\prime \prime}$ & $21^{\circ} 0^{\prime} 36.90^{\prime \prime}$ & EU01 & dirt-road & dirt-road & dirt-road \\
\hline
\end{tabular}


DOI: 10.34101/ACTAAGRAR/1/8495

Table 1. continued

\begin{tabular}{|c|c|c|c|c|c|c|}
\hline \multirow{2}{*}{$\begin{array}{c}\text { Sampling } \\
\text { site }\end{array}$} & \multirow{2}{*}{ GPS: $\mathbf{N}$} & \multirow{2}{*}{ GPS: E } & \multirow{2}{*}{$\begin{array}{c}\text { UTM } \\
(10 \times 10 \mathrm{~km})\end{array}$} & \multicolumn{3}{|c|}{ Habitat } \\
\hline & & & & 2018 & 2019 & 2020 \\
\hline 16 & $47^{\circ} 58^{\prime} 56.58^{\prime \prime}$ & $21^{\circ} 3^{\prime} 19.58^{\prime \prime}$ & EU01 & pasture & pasture & pasture \\
\hline 17 & $47^{\circ} 59^{\prime} 18.88^{\prime \prime}$ & $21^{\circ} 4^{\prime} 4.88^{\prime \prime}$ & EU00 & pasture & pasture & pasture \\
\hline 18 & $47^{\circ} 59^{\prime} 20.28^{\prime \prime}$ & $21^{\circ} 4^{\prime} 8.32^{\prime \prime}$ & EU01 & stubble & & \\
\hline 19 & $47^{\circ} 59^{\prime} 13.44 "$ & $21^{\circ} 4^{\prime} 20.34^{\prime \prime}$ & EU01 & hayfield & hayfield & hayfield \\
\hline 20 & $47^{\circ} 59^{\prime} 15.54 "$ & $21^{\circ} 4^{\prime} 30.84^{\prime \prime}$ & EU01 & hayfield & hayfield & wheat field \\
\hline 25 & $47^{\circ} 57^{\prime} 53.70^{\prime \prime}$ & $21^{\circ} 4^{\prime} 51.62^{\prime \prime}$ & EU01 & red clover field & ruderal site & weedy red clover field \\
\hline 26 & $47^{\circ} 57^{\prime} 49.78^{\prime \prime}$ & $21^{\circ} 4^{\prime} 44.69^{\prime \prime}$ & EU01 & dirt-road & & \\
\hline 27 & $47^{\circ} 57^{\prime} 50.82^{\prime \prime}$ & $21^{\circ} 4^{\prime} 57.53^{\prime \prime}$ & EU01 & red clover field & weedy alfalfa field & weedy red clover field \\
\hline 28 & $47^{\circ} 57^{\prime} 50.52^{\prime \prime}$ & $21^{\circ} 4^{\prime} 29.04^{\prime \prime}$ & EU01 & red clover field & weedy alfalfa field & weedy red clover field \\
\hline 29 & $47^{\circ} 57^{\prime} 50.40^{\prime \prime}$ & $21^{\circ} 4^{\prime} 22.02^{\prime \prime}$ & EU01 & alfalfa field & alfalfa field & alfalfa field \\
\hline 30 & $47^{\circ} 55^{\prime} 55.15^{\prime \prime}$ & $21^{\circ} 1^{\prime} 12.29^{\prime \prime}$ & EU00 & roadside & roadside & roadside \\
\hline 31 & $47^{\circ} 56^{\prime} 12.18^{\prime \prime}$ & $21^{\circ} 1^{\prime} 0.06^{\prime \prime}$ & EU00 & stubble & sunflower field & maize field \\
\hline 32 & $47^{\circ} 56^{\prime} 16.31 "$ & $21^{\circ} 1^{\prime} 0.43^{\prime \prime}$ & EU00 & stubble & maize field & wheat field \\
\hline 33 & $47^{\circ} 56^{\prime} 8.16^{\prime \prime}$ & $21^{\circ} 0^{\prime} 43.20^{\prime \prime}$ & EU00 & roadside & roadside & roadside \\
\hline 34 & $47^{\circ} 56^{\prime} 53.69^{\prime \prime}$ & $21^{\circ} 0 ' 35.04^{\prime \prime}$ & EU01 & roadside & roadside & roadside \\
\hline 35 & $47^{\circ} 57^{\prime} 26.77^{\prime \prime}$ & $21^{\circ} 0^{\prime} 54.90^{\prime \prime}$ & EU01 & hayfield & hayfield & hayfield \\
\hline 36 & $47^{\circ} 57^{\prime} 26.57^{\prime \prime}$ & $21^{\circ} 0^{\prime} 57.67^{\prime \prime}$ & EU01 & stubble & maize field & maize field \\
\hline 37 & $47^{\circ} 56^{\prime} 49.26^{\prime \prime}$ & $21^{\circ} 2^{\prime} 10.85^{\prime \prime}$ & EU01 & stubble & wheat field & maize field \\
\hline 38 & $47^{\circ} 56^{\prime} 49.28^{\prime \prime}$ & $21^{\circ} 1^{\prime} 59.42^{\prime \prime}$ & EU01 & stubble & maize field & maize field \\
\hline 39 & $47^{\circ} 56^{\prime} 49.86^{\prime \prime}$ & $21^{\circ} 2^{\prime} 1.33^{\prime \prime}$ & EU01 & roadside & roadside & roadside \\
\hline 40 & $47^{\circ} 56^{\prime} 42.05^{\prime \prime}$ & $21^{\circ} 1^{\prime} 56.09^{\prime \prime}$ & EU01 & alfalfa field & alfalfa field & stubble \\
\hline
\end{tabular}

Table 2. Orthoptera sampling sites of the formerly published investigations in the surroundings of the studied area in the firth region of Sajó and Tisza rivers

\begin{tabular}{|c|c|c|c|c|c|c|}
\hline $\begin{array}{c}\text { Sampling } \\
\text { site }\end{array}$ & $\begin{array}{c}\text { UTM } \\
(10 \times 10 \text { km })\end{array}$ & $\begin{array}{c}\text { UTM } \\
(2.5 \times 2.5 \mathrm{~km}) \\
\end{array}$ & Township & Date & $\begin{array}{c}\text { Source } \\
\text { code }\end{array}$ & Habitat \\
\hline 41 & DU90 & B2 & Igrici & 1972 & $\mathrm{R}$ & sandy grassland \\
\hline 42 & DU90 & B2 & Igrici & 1973 & $\mathrm{R}$ & sandy grassland \\
\hline 43 & DU90 & B2 & Igrici & 1974 & $\mathrm{R}$ & sandy grassland \\
\hline 44 & EU01 & B2 & Kesznyéten & 1993 & G & moved meadow \\
\hline 45 & EU01 & B2 & Kesznyéten & 1993 & $\mathrm{~N}$ & \\
\hline 46 & EU01 & $\mathrm{B} 2$ & Kesznyéten & 1994 & G & moved meadow \\
\hline 47 & EU01 & B2 & Kesznyéten & 1994 & $\mathrm{~N}$ & \\
\hline 48 & EU00 & D4 & Polgár & 1908 & $\mathrm{~J}$ & \\
\hline 49 & DU91 & $\mathrm{C} 2$ & Sajóhidvég & 1993 & $\mathrm{~N}$ & \\
\hline 50 & DU91 & $\mathrm{C} 2$ & Sajóhidvég & 1994 & $\mathrm{~N}$ & \\
\hline 51 & DU91 & $\mathrm{C} 2$ & Sajóhídvég & 1993 & G & stubble \\
\hline 52 & DU91 & $\mathrm{C} 2$ & Sajóhídvég & 1994 & G & stubble \\
\hline
\end{tabular}

Source codes refer to the published sources 

(yellow dots), and the $10 \times 10 \mathrm{~km}$ UTM cells covering the studied area and its surroundings

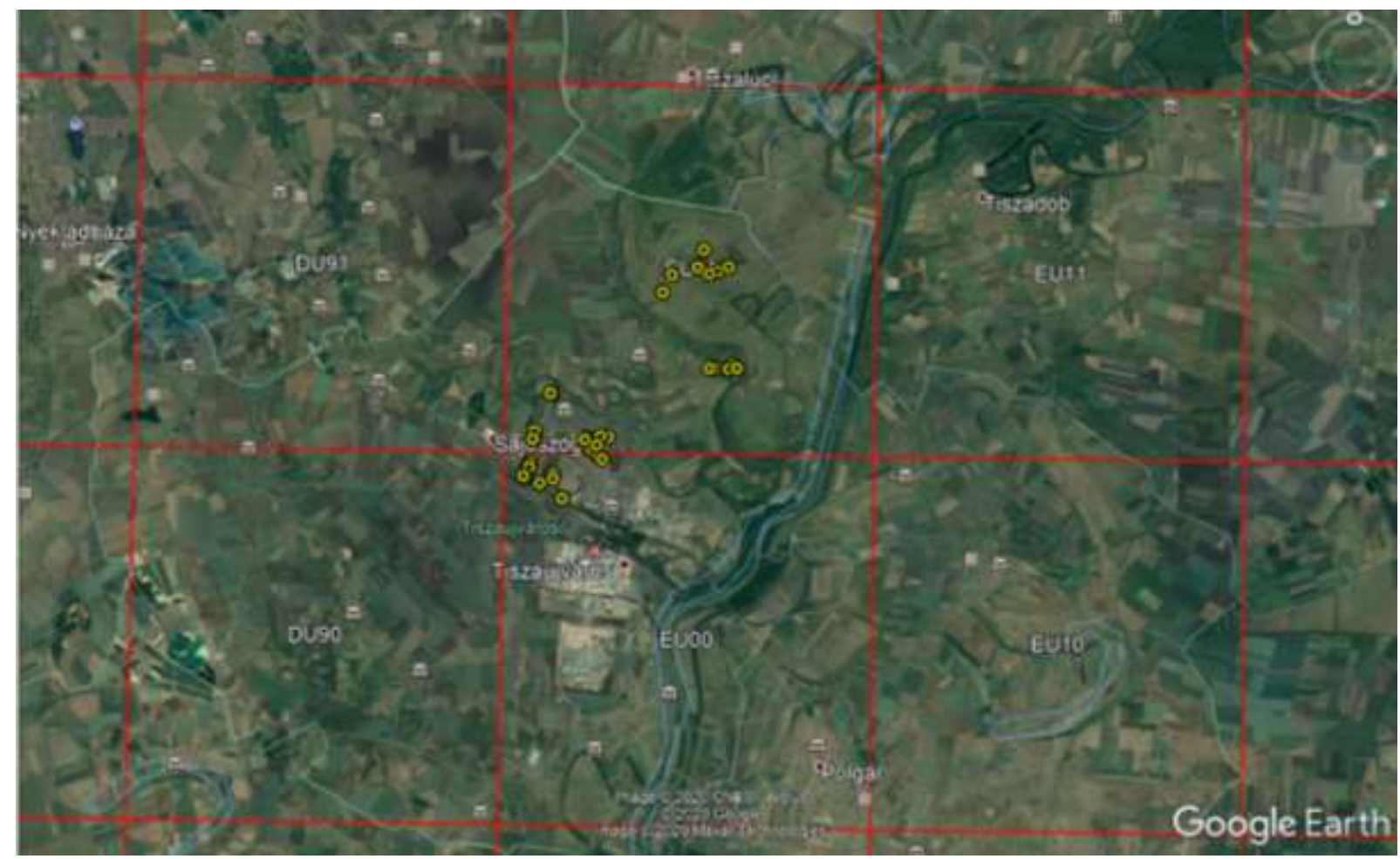

Source: GoogleEarth 2020

\section{RESULTS AND DISCUSSION}

\section{Distribution data of Orthoptera species}

Phaneroptera nana (Fieber, 1853)

19/2020 hayfield, 20/2020 wheat field, 41/1972[R]

\section{Leptophyes albovittata (Kollar, 1833)}

7/2019 dirt-road, 7/2020 dirt-road, 18/2018 stubble, 19/2018 hayfield, 19/2019 hayfield, 20/2019 hayfield, 33/2019 roadside

\section{Conocephalus fuscus (Fabricius, 1793)}

1/2020 stubble, 5/2019 wheat field, 6/2020 dirtroad, 7/2018dirt-road, 7/2019 dirt-road, 7/2020dirtroad, 9/2018dirt-road, 9/2019 dirt-road, 13/2019 alfalfa field, 14/2018 sunflower field, 15/2019 dirt-road, 19/2019 hayfield, 19/2020 hayfield, 21/2019 wheat field, 21/2020 wheat field, 28/2019 weedy alfalfa field, $30 / 2019$ roadside, 33/2019 roadside, 33/2020 roadside, $35 / 2018$ hayfield, $35 / 2020$ hayfield, $39 / 2020$ roadside

\section{Ruspolia nitidula (Scopoli, 1786)}

9/2020 dirt-road, 15/2020 dirt-road, 19/2018hayfield, 19/2020 hayfield, 20/2019 hayfield, 21/2019 wheat field, 30/2020 roadside, 31/2018 roadside, 34/2020 roadside, 35/2018hayfield, 35/2020hayfield

\section{Tettigonia viridissima (Linnaeus, 1758)}

5/2018 alfalfa field, 6/2020 dirt-road, 14/2019 wheat field, 19/2019 hayfield, 19/2020 hayfield, 20/2019 hayfield, 21/2019 wheat field, 25/2018 red clover field, 31/2019 sunflower field
Platycleis grisea (Fabricius, 1781)

44/1993[G], 45/1993[N], 46/1994[G], 47/1994[N]

Platycleis affinis (Fieber, 1853)

4/2018 stubble, 6/2018 dirt-road, 7/2018 dirt-road, 9/2018 dirt-road, 43/1974[R]

Tessellana veyseli (Koçak, 1984)

$8 / 2018$ wheat field

\section{Montana montana (Kollar, 1833)} 43/1974[R]

\section{Bicolorana bicolor (Philippi, 1830)}

9/2020 dirt-road, 17/2019 pasture, 30/2018 roadside, 33/2018 roadside, 33/2019 roadside, 33/2020 roadside, 35/2018 hayfield

\section{Roeseliana roeseli (Hagenbach, 1822)}

2/2018 dirt-road, 5/2019 wheat field, 6/2019 dirtroad, 7/2019 dirt-road, 7/2020 dirt-road, 9/2019 dirtroad, 9/2020 dirt-road, 11/2019 wheat field, 13/2020 alfalfa field, 15/2019 wheat field, 15/2020 dirt-road, $16 / 2018$ pasture, $16 / 2019$ pasture, $17 / 2018$ pasture, $17 / 2019$ pasture, 18/2018 stubble, 19/2018 hayfield, 19/2019 hayfield, 19/2020 hayfield, 20/2018 hayfield, 20/2019 hayfield, 20/2020 wheat field, 21/2018 hayfield, 21/2019 wheat field, 23/2019 pasture, 25/2019 ruderals, 28/2019 weedy alfalfa field, 29/2019 alfalfa field, 30/2019 roadside, 30/2020 roadside, $32 / 2020$ wheat field, 33/2019 roadside, 34/2019 roadside, 35/2018 hayfield, 35/2019 hayfield, 35/2020 hayfield, 37/2019 wheat field 


\section{Gampsocleis glabra (Herbst, 1786)}

9/2019 dirt-road, 13/2018 alfalfa field, 16/2019 pasture, 19/2019 hayfield, 20/2020 wheat field, 22/2019 pasture, 23/2019 pasture, 24/2018red clover field,24/2020red clover field

\section{Gryllus campestris (Linnaeus, 1758)}

3/2018 sunflower field, 4/2018 stubble, 10/2018 stubble, 10/2019 maize field than stubble, 13/2018 alfalfa field, 28/2020 weedy red clover field, 32/2019 maize field

\section{Melanogryllus desertus (Pallas, 1771)}

28/2018 red clover field, 35/2019 hayfield, 36/2018 stubble, 44/1993[G], 45/1993[N], 46/1994[G], 47/1994[N]

\section{Oecanthus pellucens (Scopoli, 1763)}

14/2018 sunflower field, 17/2018 pasture, 19/2019 hayfield, 20/2019 hayfield, 37/2019 wheat field, 39/2018 roadside

\section{Calliptamus italicus (Linnaeus, 1758)}

2/2018 dirt-road, 4/2018 stubble, 4/2020 stubble, 5/2018 alfalfa field, 5/2019 wheat field, 6/2018dirtroad, 6/2019dirt-road, 9/2018dirt-road, 9/2019dirtroad, 9/2020dirt-road, 10/2018 stubble, 11/2018 stubble, 11/2019 wheat field, 13/2018alfalfa field, 13/2019 alfalfa field, 13/2020alfalfa field, 14/2019 wheat field, 15/2018dirt-road, 15/2019 dirt-road, 15/2020dirt-road, 18/2018 stubble, 21/2018 hayfield, 24/2018 red clover field, 25/2018 red clover field, 25/2020 weedyred clover field, 27/2020 weedyred clover field, 28/2019 weedyalfalfa field, 29/2019 alfalfa field, 30/2018 roadside, 30/2019 roadside, 30/2020 roadside, 32/2020 wheat field, 33/2018 roadside, 34/2018 roadside, 34/2019 roadside, 34/2020 roadside, 37/2018 stubble, 38/2018 stubble, 38/2020 maize field, 39/2018 roadside, 39/2019 roadside, 39/2020roadside, 40/2018 alfalfa field, 40/2019 alfalfa field, 40/2020 stubble; 49/1993[N], 50/1994[N], 51/1993[G], 52/1994[G]

Celes variabilis (Pallas, 1771)

19/2019 hayfield

Oedaleus decorus (Germar, 1825) 42/1973[R], 43/1974[R]

Oedipoda caerulescens (Linnaeus, 1758) 41/1972[R], 42/1973[R], 43/1974[R]

\section{Aiolopus thalassinus (Fabricius, 1781)}

16/2018 pasture, 16/2019 pasture, 17/2018 pasture, 18/2018 stubble, 22/2018 pasture, 23/2018pasture, 23/2019 pasture, 23/2020pasture, 27/2018 red clover field, 37/2018 stubble, 41/1972[R], 44/1993[G], 45/1993[N], 6/1994[G], 47/1994[N]

\section{Mecostethus parapleurus (Hagenbach, 1822)}

15/2020 dirt-road, 17/2018 pasture, 20/2018 hayfield, 20/2019 hayfield, 24/2020 red clover field, 25/2020 weedy red clover field, 26/2018 dirt-road, 27/2018 red clover field, 27/2019 weedy alfalfa field, 27/2020 weedy red clover field, 28/2019 weedy alfalfa field, 29/2018 alfalfa field, 29/2020 alfalfa field, $35 / 2019$ hayfield, $35 / 2020$ hayfield

\section{Acrida ungarica (Herbst,1786)}

4/2018 stubble, 7/2018 dirt-road, 9/2019 dirt-road, 23/2019 pasture, 23/2020 pasture, 41/1972[R], 42/1973[R], 43/1974[R]

Chrysochraon dispar (Germar, 1834)

35/2018 hayfield, 35/2019 hayfield

Stenobothrus crassipes (Charpentier, 1825)

41/1972[R], 43/1974[R]

Stenobothrus nigromaculatus (Herrich-Schaeffer, 1840) 41/1972[R], 43/1974[R]

\section{Stenobothrus stigmaticus (Rambur, 1838)} 41/1972[R]

Omocestus rufipes (Zetterstedt, 1821)

6/2020 dirt-road, 7/2018dirt-road, 7/2019 dirt-road, 7/2020 dirt-road, 9/2018 dirt-road, 9/2020 dirt-road, 10/2018 stubble, 13/2018 alfalfa field, 15/2018 dirtroad, 15/2019 dirt-road, 15/2020 dirt-road, 16/2018 pasture, 16/2019 pasture, 16/2020 pasture, 17/2018 pasture, 17/2019 pasture, 17/2020pasture, 19/2018hayfield, 19/2019 hayfield, 19/2020 hayfield, 20/2019 hayfield, 20/2020 wheat field, 21/2018 hayfield, 21/2019 wheat field, 21/2020 wheat field, 22/2018pasture, 22/2019 pasture, 22/2020pasture, 23/2018pasture, 23/2019 pasture, 23/2020 pasture, 24/2019 ruderals, 25/2020 weed yred clover field, 27/2019 weedy alfalfa field, 27/2020 weedy red clover field, 28/2019 weedy alfalfa field, 29/2019 alfalfa field, 29/2020 alfalfa field, 30/2018 roadside, 30/2019 roadside, 30/2020 roadside, 33/2018 roadside, 33/2019 roadside, 33/2020 roadside, 34/2018 roadside, 34/2019 roadside, 34/2020 roadside, 35/2019 hayfield, 35/2020 hayfield, 39/2018 roadside, 39/2019 roadside, 39/2020 roadside, 40/2019 alfalfa field, 41/1972[R], 42/1973[R], 43/1974[R]

\section{Omocestus haemorrhoidalis (Charpentier, 1825)}

2/2018 dirt-road, 4/2018 stubble, 6/2019, dirt-road, 7/2018 dirt-road, 7/2019 dirt-road, 9/2018 dirt-road, 9/2019dirt-road, 10/2018 stubble, 11/2018 stubble, 13/2018 alfalfa field, 13/2019alfalfa field, 14/2018 stubble, 14/2019 wheat field, 15/2018dirt-road, 15/2019dirt-road, 16/2020 pasture, 17/2018 pasture, 21/2018 hayfield, 24/2018 red clover field, 24/2019 ruderals, 25/2018 red clover field, 25/2019 ruderals, 27/2018 red clover field, 27/2019 weedy alfalfa field, 28/2018 red clover field, 28/2019 weedy alfalfa field, 30/2018 roadside, 30/2020roadside, 31/2018 stubble, $33 / 2018$ roadside, $34 / 2018$ roadside, 35/2018 hayfield, 37/2018 stubble, 38/2018 stubble, 39/2018 roadside, 40/2018 alfalfa field, 41/1972[R], 43/1974[R], 44/1993[G], 45/1993[N], 46/1994[G], 47/1994[N]

Omocestus petraeus (Brisout de Barneville, 1856) 43/1974[R]

\section{Chorthippus biguttulus (Linnaeus, 1758)}

6/2019 dirt-road, 7/2019 dirt-road, 10/2018 stubble, 16/2019 pasture, 32/2018 stubble, 33/2018 roadside, 34/2019roadside, 34/2020roadside, 35/2019 hayfield, 37/2019 wheat field, 39/2020 roadside, 41/1972[R], 42/1973[R], 43/1974[R] 


\section{Chorthippus brunneus (Thunberg, 1815)}

$1 / 2018$ stubble, $1 / 2020$ stubble, $3 / 2019$ rape field, 4/2018 stubble, 4/2020 stubble, 5/2019 wheat field, 6/2018 dirt-road, 6/2019 dirt-road, 6/2020 dirt-road, 7/2018 dirt-road, 7/2019 dirt-road, 7/2020 dirt-road, 9/2018 dirt-road, 9/2019 dirt-road, 9/2020 dirt-road, $11 / 2019$ wheat field, 13/2018 alfalfa field, 13/2019 alfalfa field, 13/2020 alfalfa field, 14/2018 stubble, $14 / 2019$ wheat field, 15/2018 dirt-road, 15/2019 dirtroad, 15/2020 dirt-road, 21/2019 wheat field, 21/2020 wheat field, 24/2019 ruderals, 24/2020 red clover field, 25/2019 ruderals, 25/2020 weedy red clover field, $27 / 2018$ red clover field, 27/2020 weedy red clover field, 28/2020 weedy red clover field, 29/2020 alfalfa field, 30/2019 roadside, 30/2020 roadside, 31/2018 stubble, 32/2020 wheat field, 33/2019 roadside, $34 / 2018$ roadside, $34 / 2019$ roadside, $34 / 2020$ roadside, $35 / 2018$ hayfield, $35 / 2019$ hayfield, $35 / 2020$ hayfield, $38 / 2018$ stubble, 39/2019 roadside, 39/2020 roadside, $39 / 2018$ roadside, 40/2019 alfalfa field, 40/2020 stubble, 41/1972[R],42/1973[R], 43/1974[R]

\section{Chorthippus mollis (Charpentier, 1825)} 41/1972[R], 43/1974[R]

\section{Chorthippus dorsatus (Zetterstedt,1821)}

2/2018 dirt-road, 5/2019 wheat field, 6/2018 dirtroad, 6/2019 dirt-road, 6/2020 dirt-road, 7/2018 dirtroad, 7/2020 dirt-road, 9/2018 dirt-road, 9/2019 dirtroad, 9/2020 dirt-road, 10/2018 stubble, 13/2018 alfalfa field, 13/2019 alfalfa field, 13/2020 alfalfa field, $14 / 2019$ wheat field, 15/2018 dirt-road, 15/2019 dirtroad, 15/2020 dirt-road, 16/2018 pasture, 16/2019 pasture, 16/2020 pasture, 17/2018 pasture, 17/2019 pasture, 17/2020 pasture, 18/2018 stubble, 19/2018 hayfield, 19/2019 hayfield, 19/2020 hayfield, 20/2018 hayfield, 20/2019 hayfield, 21/2018 hayfield, 21/2020 wheat field, 22/2020 pasture, 23/2019 pasture, 23/2020 pasture, 24/2018 red clover field, 24/2019 ruderals, 24/2020 red clover field, 25/2019 ruderals, 26/2018 dirt-road, 27/2018 red clover field, 27/2020 weedy red clover field, 28/2019 weedy alfalfa field, 29/2020 alfalfa field, 30/2018 roadside, 30/2019 roadside, $30 / 2020$ roadside, 33/2018 roadside, 33/2019 roadside, $33 / 2020$ roadside, $34 / 2018$ roadside, 34/2019 roadside, $34 / 2020$ roadside, $35 / 2018$ hayfield, $35 / 2019$ hayfield, $35 / 2020$ hayfield, 37/2018 stubble, 39/2019 roadside, 39/2020 roadside, 40/2019 alfalfa field, 41/1972[R], 43/1974[R], 44/1993[G], 45/1993[N], 46/1994[G], 47/1994[N]

\section{Chorthippus loratus (Fischer von Waldheim, 1846)}

43/1974[R] * Revised by Nagy 2003.

\section{Chorthippus dichrous (Eversmann, 1859)}

9/2020 dirt-road, 13/2019 alfalfa field, 17/2018 pasture, 19/2018 hayfield, 19/2020 hayfield, 20/2018 hayfield, 20/2020 wheat field, 21/2018 hayfield, $21 / 2020$ wheat field, 26/2018 dirt-road, 27/2018 red clover field, 27/2019 weedy alfalfa field, 28/2018 red clover field, 30/2018 roadside, 33/2018 roadside, 35/2018hayfield, 35/2020hayfield, 39/2018 roadside, $43 / 1974[\mathrm{R}]$
Chorthippus oschei (Helversen, 1986) (formerly published as $C$. albomarginatus (DeGeer, 1773))

4/2018 stubble, 6/2018 dirt-road, 7/2018 dirt-road, 9/2018dirt-road, 9/2019dirt-road, 10/2018 stubble,13/2018 alfalfa field, 15/2018 dirt-road, $16 / 2018$ pasture, $16 / 2020$ pasture, $17 / 2018$ pasture, $17 / 2020$ pasture, 19/2018 hayfield, 19/2019 hayfield, 19/2020 hayfield, 20/2018 hayfield, 20/2019hayfield, $21 / 2018$ hayfield, $23 / 2018$ pasture, 23/2019 pasture, 23/2020 pasture, 29/2018 alfalfa field, 30/2018 roadside, 33/2018 roadside, 34/2018 roadside, 35/2018 hayfield, 39/2018 roadside, 43/1974[R]

\section{Pseudochorthippus parallelus (Zetterstedt, 1821)}

2/2018 dirt-road, 6/2020 dirt-road, 7/2018 dirt-road, 7/2020 dirt-road, 9/2018 dirt-road, 9/2019 dirt-road, 9/2020 dirt-road, 11/2019 wheat field, 13/2020 alfalfa field, 15/2019 dirt-road, 15/2020 dirt-road, 16/2018 pasture, 16/2019 pasture, 16/2020 pasture, 17/2018 pasture, 17/2019 pasture, 17/2020 pasture, 19/2018 hayfield, 19/2019 hayfield, 19/2020 hayfield, 20/2018 hayfield, 20/2019 hayfield, 20/2020 wheat field, 21/2018 hayfield, 21/2019 wheat field, 22/2018 pasture, 22/2019 pasture, 23/2019 pasture, 24/2020 red clover field, 25/2019 ruderals, 25/2020 weedy red clover field, 26/2018 dirt-road, 28/2019 weedy alfalfa field, 29/2018 alfalfa field, 29/2020 alfalfa field, $30 / 2018$ roadside, $30 / 2019$ roadside, $30 / 2020$ roadside, $33 / 2018$ roadside, 33/2019 roadside, 33/2020 roadside, $34 / 2018$ roadside, $34 / 2019$ roadside, 34/2020 roadside, $35 / 2018$ hayfield, 35/2019 hayfield, 35/2020 hayfield, $37 / 2018$ stubble

Euchorthippus declivus (Brisout de Barneville, 1848)

2/2018 dirt-road, 6/2018 dirt-road, 7/2018 dirt-road, 7/2020 dirt-road, 9/2018 dirt-road, 9/2019 dirt-road, 9/2020 dirt-road, 13/2018 alfalfa field, 15/2018 dirtroad, 15/2020 dirt-road, 16/2018 pasture, 17/2018 pasture, 19/2018 hayfield, 20/2018 hayfield, 21/2018 hayfield, 23/2019 pasture, 29/2019 alfalfa field, $30 / 2018$ roadside, $30 / 2020$ roadside, 33/2018 roadside, $33 / 2019$ roadside, $33 / 2020$ roadside, 34/2019 roadside, $35 / 2018$ hayfield, $35 / 2019$ hayfield, $35 / 2020$ hayfield, 37/2018 stubble, 39/2018 roadside, 43/1974[R]

Euchorthippus pulvinatus (Fischer de Waldheim, 1846)

$$
\text { 41/1972[R] }
$$

Myrmeleotettix maculatus (Thunberg, 1815) 41/1972[R], 42/1973[R]

Dociostaurus maroccanus (Thunberg, 1815) 48/1908[J]

Dociostaurus brevicollis (Eversmann, 1848) $17 / 2019$ pasture, 23/2019 pasture, 23/2020 pasture, 41/1972[R], 43/1974[R]

Tetrix depressa (Brisout de Barneville, 1848) 29/2020 alfalfa field

Tetrix subulata (Linnaeu, 1758) 46/1994[G], 47/1994[N] 
In the 4 published sources data of 12 sampling sites could be found. These sources mentioned 29 Orthoptera species in the study area and its surroundings. It means that only 69 data records of orthopterans were available from this area before our investigations. The occurrence of Chorthippus loratus in Hungary was revised by Nagy (2003), and data of Chorhippus albomarginatus should be added to newly recorded $C$. oschei based on Orczi 2002. Thus, the revised list of formerly published occurrences contains 28 species.

During our 3-year study, 2241 specimens of 30 Orthoptera species were sampled in the 40 sampling sites of agricultural landscape and most of the sampled specimens were adults (1762) which could be identified at species level. With this large amount of data, we provide 540 new distribution data records of orthopterans that means an almost eightfold increase in the number of data records. It showed that this area was a relatively poorly known part of the lowland and that the agricultural lands are very poorly represented in faunistical and different entomological studies.

The number of the formerly known and newly sampled species was nearly equal, but there were only 15 species that appeared in both checklists. It showed that in the former studies agricultural land was not studied or was poorly represented. Only Garay (1995) discuss data of Calliptamus italicus population living in stubble fields where we also detected this species sometimes with large abundance (Arnóczkyné et al., 2020).

13 species mentioned only in published sources are Platycleis grisea, Montana montana, Oedaleus decorus, Oedipoda caerulescens, Stenobothrus crassipes, S. nigromaculatus, S. stigmaticus, Omocestus petraeus, Chorthippus mollis, Euchorthippuspulvinatus, Myrmeleotettix maculatus, Dociostaurus maroccanus and Tetrix subulata. Most of them are characteristic mainly for open sandy grasslands which habitat type was not investigated in recent study.

In case of newly studied habitats, we could record 15 new species compared to published data: Leptophyes albovittata, Conocephalus fuscus, Ruspolia nitidula, Tettigonia viridissima, Tessellana veyseli, Bicolorana bicolor, Roeseliana roeselii, Gampsocleisglabra, Gryllus campestris, Oecanthus pellucens, Celes variabilis, Mecostethus parapleurus, Chrysocraon dispar, Pseudochorthippus parallelus and Tetrix depressa. Many of them can be characteristic to mesic and in some cases to humid habitats that can explain differences of former and newly recorded species lists.

First and last the actualized revised Orthoptera fauna of the studied area and its surroundings contain 43 Orthoptera species that is about a third of the Hungarian Orthoptera fauna.

In this area, three protected species, Gampsocleis glabra, Acrida ungarica and Celes variabilis can be found while most of the species are common and even abundant in Hungary especially in lowlands. According to Nagy and Rácz (2007) Montana montana,
Melanogryllus desertus, Chorthippus dichrous, Myrmeleotettix maculatus, Dociostaurus maroccanus and Tetrix depressa are scattered, while Ruspolia nitidula, Gampsocleis glabra, Gryllus campestri, Celes variabilis, Mecostethus parapleurus and Euchorthippus pulvinatus are rare in Hungary, based on their spatial constancy. In the studied sites G. glabra, $M$. parapleurus and $C$. dichrous showed larger frequency than at country level. Among them G. glabra characteristic for mesic tall grasslands of lowlands, $M$. parapleurus prefer high dense vegetations of meadows and wet grasslands while $C$. dichrous also distributed mainly in the lowlands that can explain their higher local frequencies.

\section{Orthopterans of different habitat types}

Orthoptera assemblages of different habitat types showed significant differences considering both their species richness and composition. The most speciesrich habitats were hayfields and dirt-roads, while wheat fields also showed relatively large species diversity. In these habitats, at least half of the recorded species occurred. Species richness of alfalfa fields, roadsides, stubble fields, and pastures were both larger than 10 species/site. In contrast in sunflower fields, the number of caught species was 4 while in the also intensively cultivated maize and rape fields only 1-1 species could be found (Table 3). In case of the rape seed field, only one sampling was carried out in 2019 (sampling site 3), but it can only partly explain the especially low species diversity.

Weedy sites of red clover and alfalfa fields were characterized separately from properly treated ones. In degraded sites can maintain less diverse Orthoptera assemblages especially in case of the alfalfa field, where changing of vegetation structure decrease the total number of species from 14 to 8 species (Table 3 ). In ruderals and weedy fields mainly widely distributed generalists could be found such as Chorthippus dorsatus, Pseudochorthippus parallelus, Calliptamus italicus and Omocestus rufipes, while less common species of these habitat types e.g. Gampsocleis glabra, Tettigonia viridissima, Chorthippus oschei and Chorthippus biguttulus were absent in these sites.

The more widely distributed species of the studied region were Calliptamus italicus, Chorthippus dorsatus, Pseudochorthippus parallelus, Chorthippus brunneus, Omocestus haemorrhoidalis and Omocestus rufipes. One third of the caught species could be found more than half of the studied habitat types, while the other third of the species occupied up to 3 from the studied 14 habitat types (Table 3). These latest mentioned species are less common also in the fauna of the Hungarian lowland and the whole country. These locally moderately rare and rare species could be found in non-cultivated and extensively used habitats such as hayfields, pastures, alfalfa, and red clover fields. Cultures with intense cultivation and intensive use of agrochemicals had only species-poor orthopteran assemblages. 
Table 3. List of the Orthoptera species sampled between 2018 and 2020 in the firth region of the Sajó and Tisza rivers by the sampled agricultural habitat types. Species are sorted by the decreasing number of occupied habitat types, while habitat types are sorted based on their decreasing cumulated species richness

\begin{tabular}{|c|c|c|c|c|c|c|c|c|c|c|c|c|c|c|c|}
\hline & $\begin{array}{l}\overrightarrow{\overrightarrow{0}} \\
\stackrel{\overrightarrow{0}}{0} \\
\stackrel{0}{2}\end{array}$ & 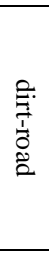 & 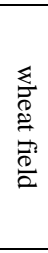 & $\begin{array}{l}\stackrel{0}{D} \\
\stackrel{\vec{D}}{D} \\
\vec{D} \\
\stackrel{\vec{D}}{2}\end{array}$ & 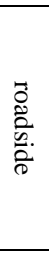 & $\frac{\mathscr{n}}{\underset{\tilde{J}}{\sigma}}$ & 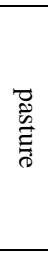 & $\begin{array}{l}\overrightarrow{0} \\
\stackrel{0}{0} \\
\stackrel{0}{0} \\
0 \\
\stackrel{0}{0} \\
\overrightarrow{0} \\
\stackrel{0}{0}\end{array}$ & 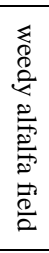 & 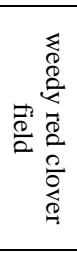 & $\begin{array}{l}\vec{\Xi} \\
\frac{0}{0} \\
\frac{0}{\infty}\end{array}$ & 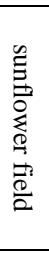 & 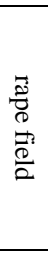 & $\begin{array}{l}\vec{\Xi} \\
\stackrel{0}{0} . \\
0 \\
\vec{D} \\
\stackrel{\vec{D}}{D}\end{array}$ & 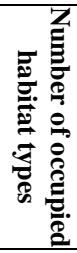 \\
\hline Chorthippus dorsatus & 1 & 1 & 1 & 1 & 1 & 1 & 1 & 1 & 1 & 1 & 1 & & & & 11 \\
\hline Pseudochorthippus parallelus & 1 & 1 & 1 & 1 & 1 & 1 & 1 & 1 & 1 & 1 & 1 & & & & 11 \\
\hline Calliptamus italicus & 1 & 1 & 1 & 1 & 1 & 1 & & 1 & 1 & 1 & & & & 1 & 10 \\
\hline Chorthippus brunneus & 1 & 1 & 1 & 1 & 1 & 1 & & 1 & & 1 & 1 & & 1 & & 10 \\
\hline Omocestus haemorrhoidalis & 1 & 1 & 1 & 1 & 1 & 1 & 1 & 1 & 1 & & 1 & & & & 10 \\
\hline Omocestus rufipes & 1 & 1 & 1 & 1 & 1 & 1 & 1 & & 1 & 1 & 1 & & & & 10 \\
\hline Roeseliana roeseli & 1 & 1 & 1 & 1 & 1 & 1 & 1 & & 1 & & 1 & & & & 9 \\
\hline Chorthippus dichrous & 1 & 1 & 1 & 1 & 1 & & 1 & 1 & 1 & & & & & & 8 \\
\hline Conocephalus fuscus & 1 & 1 & 1 & 1 & 1 & 1 & & & 1 & & & 1 & & & 8 \\
\hline Mecostethus parapleurus & 1 & 1 & & 1 & & & 1 & 1 & 1 & 1 & & & & & 7 \\
\hline Chorthippus oschei & 1 & 1 & & 1 & 1 & 1 & 1 & & & & & & & & 6 \\
\hline Euchorthippus declivus & 1 & 1 & & 1 & 1 & 1 & 1 & & & & & & & & 6 \\
\hline Gampsocleis glabra & 1 & 1 & 1 & 1 & & & 1 & 1 & & & & & & & 6 \\
\hline Chorthippus biguttulus & 1 & 1 & 1 & & 1 & 1 & 1 & & & & & & & & 6 \\
\hline Tettigonia viridissima & 1 & 1 & 1 & 1 & & & & 1 & & & & 1 & & & 6 \\
\hline Gryllus campestris & & & & 1 & & 1 & & & & 1 & & 1 & & 1 & 5 \\
\hline Oecanthus pellucens & 1 & & 1 & & 1 & & 1 & & & & & 1 & & & 5 \\
\hline Bicolorana bicolor & 1 & 1 & & & 1 & & 1 & & & & & & & & 4 \\
\hline Leptophyes albovittata & 1 & 1 & & & 1 & 1 & & & & & & & & & 4 \\
\hline Ruspolia nitidula & 1 & 1 & 1 & & 1 & & & & & & & & & & 4 \\
\hline Acrida ungarica & & 1 & & & & 1 & 1 & & & & & & & & 3 \\
\hline Aiolopus thalassinus & & & & & & 1 & 1 & 1 & & & & & & & 3 \\
\hline Melanogryllus desertus & 1 & & & & & 1 & & 1 & & & & & & & 3 \\
\hline Platycleis affinis & & 1 & & & & 1 & & & & & & & & & 2 \\
\hline Tessellana veyseli & & & 1 & & & & & & & & & & & & 2 \\
\hline Celes variabilis & 1 & & & & & & & & & & & & & & 1 \\
\hline Chrysochraon dispar & 1 & & & & & & & & & & & & & & $\mathbf{1}$ \\
\hline Dociostaurus brevicollis & & & & & & & 1 & & & & & & & & 1 \\
\hline Phaneroptera nana & & & 1 & & & & & & & & & & & & $\mathbf{1}$ \\
\hline Tetrix depressa & & & & 1 & & & & & & & & & & & 1 \\
\hline Species number & 18 & 17 & 15 & 14 & 13 & 13 & 12 & 9 & 8 & 6 & 6 & 4 & 1 & 1 & \\
\hline
\end{tabular}

\section{CONCLUSIONS}

Our results drew attention to that the Orthoptera assemblages of agricultural habitats are generally poorly investigated and known. The recent 3-year study could provide large amount of faunistical data on a poorly known firth region of the Sajó and Tisza rivers containing data of three protected species (Gampsocleis glabra, Acrida ungarica and Celes variabilis). The amount of the known records was increased eightfold.

Analysis showed that the non-cultivated habitats as dirt-roads, roadsides, stubble fields, and extensive lands of hayfields and pastures can maintain speciesrich habitats in agricultural lands. The less intensive cultures e.g. alfalfa and red clover fields and even the grasses and whet field also can preserve relatively diverse Orthoptera fauna that indicates also speciesrich herbivorous insect community of these habitat types. Contrary to the intensively cultivated maize, sunflower, and rapeseed fields lost nearly their whole Orthoptera and other non-pest herbivores, thus in intensive agricultural lands even the less intensive cultures and other artificial habitats can become the last remains of former natural and semi-natural insect assemblages.

Since changes in agricultural landscape structure are driven by the economic environment, agricultural policy and climate change have further negative effects on insect diversity including diversity beneficial organisms as predators, parasites, and pollinators. Orthopterans as indicators can be used in planning and decision making both in conservation biology and landscape planning and even in the monitoring of the effect of our activities and can help us to form a more sustainable cultivation system. 


\section{REFERENCES}

Agócs, B.-Galambos, A.-Hegymegi, P.-Kary, L.-Keszthelyi, K.Kiss, A.-Kovács, V.-Néráth, M.-Rezneki, R.-Sztahura, E.Tóth, P.-Várszegi, G. (2015): Agrár-környezetgazdálkodás. Nemzeti Agrárgazdasági Kamara, Budapest, 72 p. https://www.nak.hu/kiadvanyok/ kiadvanyok/130-akgkezikonyv/file

Agrárgazdasági Kutató Intézet (2013): A magyar mezőgazdaság főbb ágazatainak helyzete, piaci kilátásai rövid és középtávon. Primerate Kft., Budapest. 172 p. (ISBN 978-963-491-5850)https://www.aki.gov.hu/ekpolc/reszletek/a:750

Arnóczkyné Jakab, D.-Szanyi, Sz.-Nagy, A. (2020): Az olasz sáska (Caelifera: Calliptamus italicus Linnaeus, 1758) - Újra célkeresztben? Növényvédelem. 56 (9): 405-411.

Batáry, P.-Orczi, K.M.-Báldi, A.-Kleijn, D.-Kisbenedek, T.-Erdős, S. (2007): Effects of local and landscape scale and cattle grazing intensity on Orthoptera assemblages of the Hungarian Great Plain. Basic and Applied Ecology. 8: 280-290. https://doi.org/10.1016/j.baae.2006.03.012

Batáry, P. (2018): Természetvédelem és agrár-környezetvédelem Európában.Göttingen, Germany: University of Goettingen, $\quad \mathrm{PhD} \quad$ thesis. http://reald.mtak.hu/1128/7/dc_1490_17_doktori_mu.pdf

Báldi, A.-Kisbenedek, T. (1997): Orthopteran assemblages as indicators of grassland naturalness in Hungary. Agriculture, Ecosystems and Environment. 66: 121-129. https://doi.org/10.1016/S0167-8809(97)00068-6

Báldi, A.-Batáry, P.-Kleijn, D. (2013): Effects of grazing and biogeographic regions on grassland biodiversity in Hungary analysing assemblages of 1200 species. Agriculture, Ecosystems and Environment. 166: 28-34. https://doi.org/10.1016/j.agee.2012.03.005

CBD (2014): Global Biodiversity Outlook 4. Secretariat of the Convention on Biological Diversity, Montréal. 155 p. (ISBN-929225-539-8) https://www.cbd.int/gbo/gbo4/publication/gbo4en-hr.pdf

Conforti, P. (2011): Looking ahead in world food and agriculture: perspectives to 2050. FAO, Paris. 539 p. (ISBN-978-92-5 106903-5) http://www.fao.org/3/i2280e/i2280e.pdf

Foley, A.J.-Navin Ramankutty, N.-Brauman, A.K.-Cassidy, E.S.Gerber, J.S.-Johnston, M.-Mueller, N.D.-O'Connell, C.-Ray, D.K.-West, P.C.-Balzer, C.-Bennett, E.M.-Carpenter, S.R.Hill, J.-Monfreda, C.-Polasky, S.-Rockström, J.-Sheehan, J.Siebert, S.-Tilman, D.-Zaks, D.P.M. (2011): Solutions for a cultivated planet. Nature. 478: 337-342. https://www.nature.com/articles/nature10452?page $=56$

Fróna, D. (2018): Globális kihívások a mezőgazdaságban. Műszaki és Menedzsment Tudományi Közlemények. 3(3): 195-205. https://doi.org/10.21791/IJEMS.2018.3.16.

Garai, A. (1995): Adatok Magyarország Orthoptera faunájához. Folia Entomologica Hungarica. 56: 231-234.

IPBES (2016): The assessment report of the Intergovernmental Science-Policy Platform on Biodiversity and Ecosystem Services on pollinators, pollination and food production. In.: Potts, S. G., Imperatriz-Fonseca, V. L., Ngo, H. T., eds. Secretariat of the Intergovernmental Science-Policy Platform on Biodiversity and Ecosystem Services. Bonn, Germany. 552 p. (ISBN 978-92-807-3567-3) https://ipbes.net/sites/default/files/downloads/pdf/2017_pollinat ion_full_report_book_v12_pages.pdf
Jablonowski, J. (1910): A nagyhortobágyi sáskairtás eredményei. Természettudományi Közlöny. 42 (509): 513-525.

Kenyeres, Z.-Bauer, N.-Nagy, B. (2004): Az Orthoptera együttesek és a habitatok változásai a Tihanyi-félszigeten az 1947. és 2001. évi felvételek alapján. Állattani Közlemények. 89 (1): 37-53. http://www.mbt-biologia.hu/gen/pro/mod/let/let_fajl_kiiras. php?i_faj_azo=212

Kenyeres, Z. (2006): Adatok a Dunántúli-középhegység egyenesszárnyú (Orthoptera) faunájának ismeretéhez II. Folia Historico Naturalia Musei Matraensis. 30: 189-201. https://matramuzeum.nhmus.hu/sites/default/files/nhmusfiles/ki advanyok/folia/vol34/07_Kenyeres_egyenesszarnyu.pdf

Kenyeres, Z. (2010): Adatok a Dunántúli-középhegység egyenesszárnyú (Orthoptera) faunájának ismeretéhez III. Folia Historico Naturalia Musei Matraensis. 34: 45-58.

Kenyeres, Z.-Rácz, I.A. (2011): A Bakonyi Természettudományi Múzeum egyenesszárnyú (Orthoptera) gyüjteménye. A Bakonyi Természettudományi Múzeum közleményei. 28: 81-104. https://library.hungaricana.hu/hu/view/SZAK_BAKO_Btmk_2 $8 / ? \mathrm{pg}=86 \&$ layout $=\mathrm{s}$

Kenyeres, Z.-Szabó, Sz.-Takács, G.-Szinetár, Cs. (2020): Orthoptera assemblages as indicators for the restoration of sand grassland networks. North-Western Journal of Zoology. 16 (1): 7-14. https://www.researchgate.net/publication/338297657_Orthoptera _assemblages_as_indicators_for_the_restoration_of_sand_grasslan d_networks

Kisbenedek, T. (1997): Egyenesszárnyúak - Orthoptera. In.: Forró, L. eds. Nemzeti Biodiverzitás-monitorozó Renszer V. - Rákok, szitakötők és egyenesszárnyúak. Magyar Természettudományi Múzeum, Budapest. 55-81. (ISBN 963-7093-48-6) http://www.termeszetvedelem.hu/_user/downloads/biomon/V. $\% 20 \mathrm{R} \%$ E1kok\%2C\%20szitak\%F6t\%F5k\%20\%E9s\%20egyene ssz\%E1rny\%FAak.pdf

Koppányi, T. (1957): Hortobágyi magfüvesek Acridioidea népeségének vizsgálata. Különlenyomat a Debreceni Mezőgazdasági Akadémia Évkönyvéböl. Mezőgazdasági Kiadó, Budapest. 309-320.

McGeoch, M. (1998): The selection, testing and application of terrestrial insects as bioindicators. Biological Reviews. 73: 181201. https://doi.org/10.1111/j.1469-185X.1997.tb00029.x

Nagy, A.-Rácz, I.A. (2007): A hazai orthoptera fauna 10 x $10 \mathrm{~km}$-es UTM alapú adatbázisa. In.: Kövics, Gy.J.-Dávid, I. eds. 12. Tiszántúli Növényvédelmi Fórum, Debrecen, 2007. október 1718. Debreceni Egyetem, Debrecen. 189-198.

Nagy, A.-Sólymos, P.-Rácz, I.A. (2007): A test on the effectiveness and selectivity of three sampling methods frequently used in orthopterological field studies. Entomologica Fennica. 18 (3): 149-159. https://doi.org/10.33338/ef.84392

Nagy, A.-Bozsó, M.-Kisfali, M.-Rácz, I.A. (2008): Data on the Orthoptera fauna of the Tisza district. Tiscia, Vegetation and Fauna of River Tisza Basin II: 1-22. https://www.researchgate.net/publication/266140492_Data_on_ the_Orthoptera_fauna_of_the_Tisza_district

Nagy, A.-Kisfali, M.-Rácz, I.A. (2009): Protected Orthoptera species of agro-ecosystems in Hungary. Journal of Agricultural Sciences. $\quad 38 \quad$ (Suppl.): $106-111$. https://www.researchgate.net/publication/268976163_Protected _Orthoptera_species_of_agro-ecosystems_in_Hungary

Nagy, B. (1943): Adatok a Tiszántul Orthoptera-faunájának ismeretéhez. Folia Entomologica Hungarica. 8: 33-44. 
Nagy, B. (1953): Adatok a magyarországi gabonaföldek Saltatorianépességének ismeretéhez. Annales Instituti Protectionis Plantarum. VI. (1951): 150-167.

Nagy, B. (1992): Role of Activity Pattern in Colonization by Orthoptera. Proceedings of the Fourt European Congress of Entomology and the XIII. Internationale Symposium für die Entomofaunistik Mitteleuropas. Gödöllő, 1991. Hungarian Natural History Museum, Budapest. 351-363.

Nagy, B. (1993): Magyarországi sáskagradációk 1993-ban. Növényvédelem. 29 (9): 403-410.

Nagy, B. (2003): A revised check-list of Orthoptera-species of Hungary supplemented by Hungarian names of grasshopper species. Folia Entomologica Hungarica. 64: 85-94. http://publication.nhmus.hu/pdf/folentom/FoliaEntHung_2003_ Vol_64_85.pdf

Noss, R.F. (1990): Indicators for Monitoring Biodiversity: A Hierarchical Approach. Conservation Biology. 4: 355-364. https://doi.org/ 10.1111/j.1523-1739.1990.tb00309.x

Orczi, K.M. (2002): Orthoptera fajcsoportok bioakusztikai és morfometriai vizsgálata (On the bioacustics and Morphology of some species-group of Orthoptera). Debrecen, Hungary: University of Debrecen, $\mathrm{PhD}$ thesis. https://dea.lib.unideb.hu/dea/bitstream/handle/2437/78983/ertek ezes.pdf?sequence $=4 \&$ isAllowed $=y$

Orthoptera Species File (Version 5.0/5.0) (2020): http://orthoptera.speciesfile.org/HomePage/Orthoptera/HomePa ge.aspx
Pearson, D.L. (1994): Selecting indicator taxa for the quantitative assesment of biodiversity. Philosophical Transactions of the Royal Society of London. Ser. B. 345: 75-79.

Rácz, I.A.-Varga, Z. (1978): Beitrage zur Kenntnis der OrthopterenFauna des Sandgebietes bei Igrici.Acta Biologica Debrecina. 15: 33-39.

Szövényi, G.-Puskás, G.-Nagy, B. (2010): A Váci Naszály hegy egyenesszárnyú (Orthoptera) faunájának értékelése. A Naszály természetrajza, Rosalia. 5: 501-520. https://www.researchgate.net/publication/282337146_A_VACI _NASZALY_HEGY_EGYENESSZARNYU_ORTHOPTERA FAUNAJANAK_ERTEKELESE

Torma, A.-Gallé, R.-Bozsó, M. (2014): Effects of habitat and landscape characteristics on the arthropod assemblages (Araneae, Orthoptera, Heteroptera) of sand grassland remnants in Southern Hungary. Agriculture, Ecosystems and Environment. 196: 42-50. https://doi.org/10.1016/j.agee.2014.06.021

Wiens, J.A. (1989): Spatial scaling in ecology. Functional Ecology. 3: 385-397. http://courses.washington.edu/esrm441/pdfs/ Wiens_1989.pdf

Zilahi-Sebess, G. (1956): Rovartani vizsgálatok Észak-Tiszántúli burgonya-földeken. Acta Universitatis Debreceniensis de Ludovico Kossuth Nominatae. 3 (2): 1-30. 